\title{
Proptose causada por adenoma pituitário gigante \\ Relato de caso
}

Proptosis caused by giant pituitary adenoma - Case report

Andréa Pereira 1

Mário Luiz Ribeiro Monteiro 2
Trabalho realizado na Divisão de Clínica Oftalmológica do Hospital das Clínicas da Faculdade de Medicina da Universidade de São Paulo - USP.

${ }^{1}$ Médica estagiária.

Professor Livre Docente.

Endereço para correspondência: Rua Ademar P. Barros, 21 sala 42 - Jundiaí (SP) CEP 13201-797

Email: apsartorelli@uol.com.br

Recebido para publicação em 16.09.2002 Aceito para publicação em 07.04.2003

\begin{tabular}{|l|}
\hline R E S U M O \\
\hline Objetivo: Relatar um caso raro de proptose unilateral associada à paralisia \\
oculomotora e cegueira causados por adenoma pituitário gigante. Méto- \\
dos: A paciente foi submetida a exame clínico e oftalmológico completo, \\
campimetria, exame de imagem por ressonância magnética e estudo his- \\
topatológico do tumor após intervenção cirúrgica. Resultados: Paciente \\
de 21 anos apresentou perda visual progressiva bilateral associada à prop- \\
tose e limitação da motilidade ocular do olho esquerdo. A campimetria re- \\
velou hemianopsia temporal completa no olho direito e havia ausência de \\
percepção luminosa no olho esquerdo. O exame de imagem por ressonância \\
magnética mostrou extenso tumor infiltrando o terceiro ventrículo, seio \\
cavernoso e ápice da órbita à esquerda. O estudo histopatológico revelou \\
se tratar de adenoma hipofisário produtor de hormônio de crescimento e \\
prolactina sem sinais de malignidade. Conclusão: Este caso é de interesse \\
não apenas pelo fato do envolvimento orbitário pelos adenomas ser ex- \\
tremamente raro, mas também para salientar a importância de diagnóstico \\
precoce uma vez que apesar da histologia benigna, os adenomas invasivos \\
nem sempre seguem curso clínico favorável.
\end{tabular}

Descritores: Exoftalmia/complicações; Neoplasias hipofisárias/cirurgia; Neoplasias hipofisárias/radioterapia; Adenoma; Campimetria/métodos; Cegueira/etiologia; Glândula pituitária/patologia; Relato de caso

\section{INTRODUÇÃO}

Adenoma hipofisário é uma afecção relativamente comum, compreendendo cerca de 7 a $17,8 \%$ dos tumores intracranianos ${ }^{(1)}$. No entanto, a variante gigante é rara, limitada a poucos casos relatados na literatura ${ }^{(2)}$. O termo adenoma pituitário gigante se aplica primariamente ao tumor que apresenta 4 centímetros ou mais de diâmetro, e que, portanto, se estende muito além dos limites da sela túrcica ${ }^{(2-3)}$, com várias manifestações neuroftálmicas associadas. Entre estas estão a perda visual por compressão da via óptica, as paralisias dos nervos oculomotor, troclear, abducente, e o comprometimento da inervação simpática ocular, por invasão do seio cavernoso, além da lesão do nervo trigêmio que pode levar a perda sensitiva na região periocular.

O objetivo deste trabalho é documentar uma paciente com a manifestação incomum de proptose unilateral associada à paralisia oculomotora e cegueira do mesmo lado, decorrente de um adenoma hipofisário gigante.

RELATO DE CASO

Paciente de 21 anos, sexo feminino, branca, foi encaminhada ao depar- 
tamento de oftalmologia, em outubro de 2000, queixando-se de perda visual bilateral progressiva, associada à proptose, ptose palpebral e limitação da motilidade ocular do olho esquerdo (OE). Referia que o quadro havia iniciado em novembro de 1994, quando começou a apresentar cefaléia holocraniana, amenorréia e galactorréia, associada com baixa visual lentamente progressiva, estrabismo divergente e ptose palpebral à esquerda. Na época procurou serviço médico onde foi diagnosticado processo expansivo da região selar, supra e paraselar, sendo submetida a craniotomia fronto-temporal esquerda com ressecção parcial do tumor, seguida de tratamento clínico, não sabendo referir sobre a medicação utilizada. Em 1996 houve recidiva tumoral, sendo acompanhada até 1998 quando começou a apresentar baixa visual à direita sendo constatado volumosa massa supraselar, subtemporal e subfrontal com invasão do seio cavernoso. O campo visual mostrava hemianopsia temporal no olho direito (OD) e ausência de percepção luminosa no OE. Foi então submetida a duas novas cirurgias com ressecção parcial do tumor. O estudo histopatológico incluindo imunohistoquímica revelou tratar-se de adenoma hipofisário produtor de hormônio de crescimento (GH) e prolactina (PRL), sem sinais de malignidade. Manteve-se com tratamento clínico com inibidores da liberação de hormônio do crescimento (octreotida) e da prolactina (bromocriptina) até 1999 quando apresentou novo aumento da massa tumoral, sendo então submetida à radioterapia no período de janeiro a março de 2000.

Ao exame oftalmológico, apresentava acuidade visual de 20/20 no OD e ausência de percepção luminosa no OE. Evidenciava-se proptose à esquerda com exoftalmometria (Hertel) de $17 \mathrm{~mm}$ no OD e $23 \mathrm{~mm}$ no OE (Figura 1). Apresentava quadro de paralisia completa do nervo oculomotor e troclear à esquerda com preservação da abdução daquele olho. O fundo de olho mostrava-se com palidez de papila em banda no OD e palidez total de papila no OE. Não apresentava alterações no segmento anterior ao exame da biomicroscopia e a tonometria de aplanação foi normal. A campimetria (manual e computadorizada)

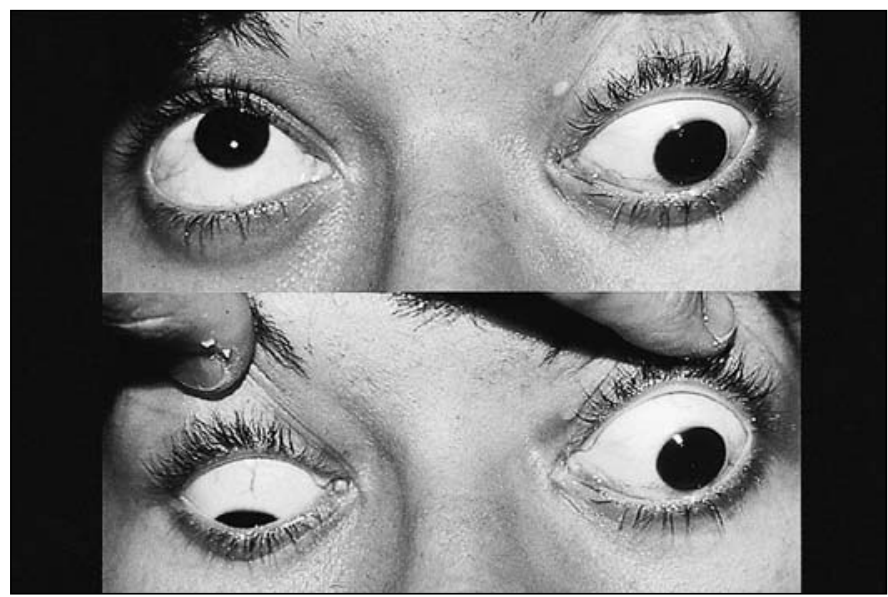

Figura 1 - Proptose e oftalmoplegia à esquerda. Acima, em supraversão; abaixo, em infraversão revelou hemianopsia temporal completa no OD, com preservação do hemicampo nasal. Apresentava à imagem por ressonância magnética, lesão tumoral hipofisária com extensão supra e paraselar, atingindo o terceiro ventrículo e seio cavernoso à esquerda, com dimensões que mediam: $6,2 \mathrm{~cm} \mathrm{X} 5,8 \mathrm{~cm} \mathrm{X}$ $5,0 \mathrm{~cm}$ (Figura 2). O tumor também se estendia até o ápice da órbita, com pequena invasão para dentro da cavidade orbitária (Figura 3).

\section{DISCUSSÃO}

Os adenomas hipofisários são lesões relativamente freqüentes, compreendendo cerca de 7 a $17,8 \%$ de todos os tumores intracranianos ${ }^{(1)}$. A maior parte dos adenomas hipofisários se situa dentro da sela túrcica, sendo denominados microadenomas e não acarretando manifestações oftálmicas. Quando possuem tamanho maior que $10 \mathrm{~mm}$ passam a ser denominados macroadenomas podendo causar manifesta-

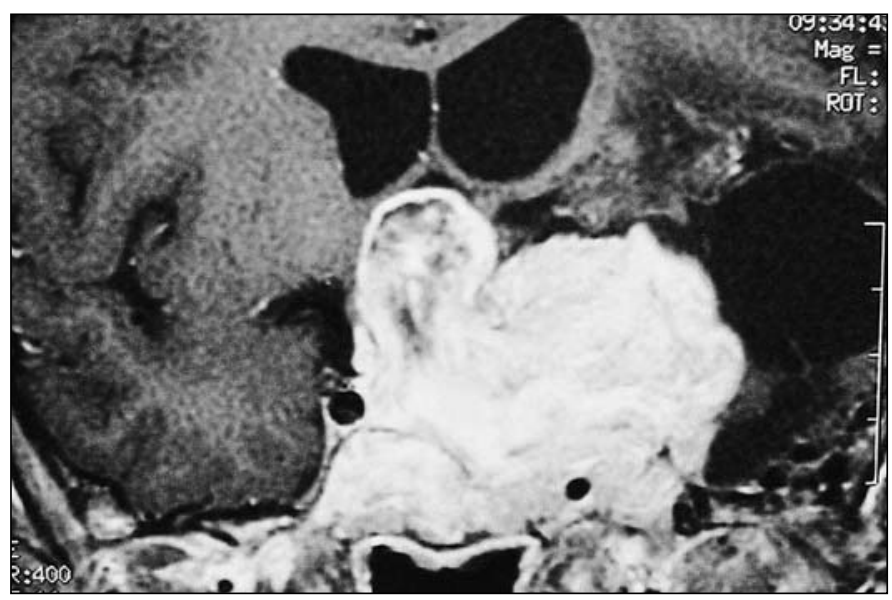

Figura 2 - Imagem por ressonância magnética em corte coronal mostrando grande tumor hipofisário com extensão supra e para selar à esquerda

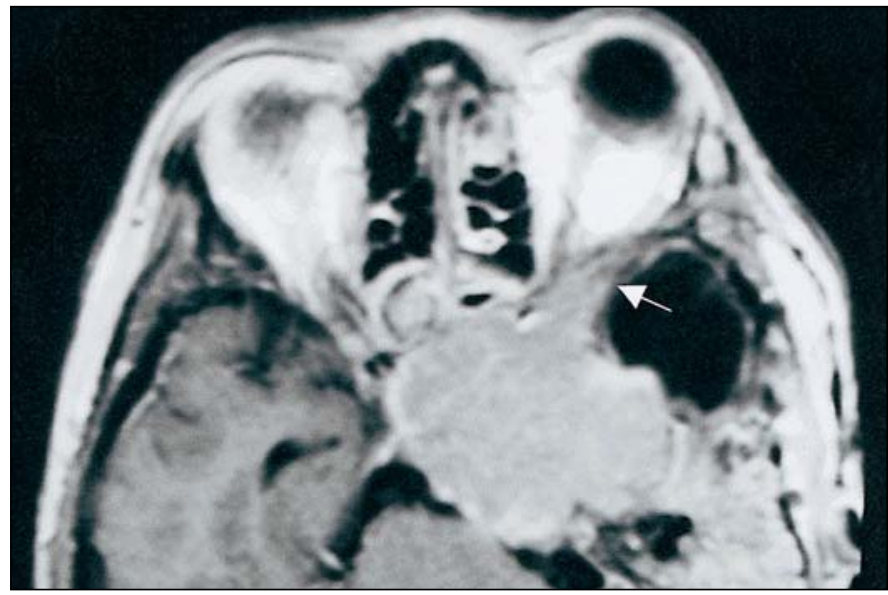

Figura 3 - Imagem por ressonância magnética, corte axial, evidenciando grande com invasão do ápice da órbita à esquerda (seta) 
ções neuroftalmológicas em decorrência da compressão de estruturas adjacentes.

O adenoma hipofisário gigante, no entanto, é uma variante pouco comum, tendo sido descrito pela primeira vez em 1940 por Jefferson, o qual relatou uma série de tumores extensos ${ }^{(4)}$. Esta denominação se refere aos tumores com quatro centímetros ou mais de diâmetro ${ }^{(2-3)}$. Os achados histológicos, não se diferenciam daqueles encontrados nos adenomas usuais, sendo, portanto, histologicamente tumores benignos, podendo ser secretantes ou não funcionantes. De um total de 21 pacientes portadores de adenoma hipofisário gigante, estudados por Grote, cerca de um terço eram portadores de prolactinomas e metade de tumores não funcionantes causando graus variados de falência hipofisária. Ainda se discute se esta variante de tumor ocorre devido ao diagnóstico realizado tardiamente, ou se ele possui um padrão de crescimento histológico diferenciado, ocorrendo de uma maneira mais invasiva e agressiva ${ }^{(3)}$.

Os tumores hipofisários quando não funcionantes, podem permanecer assintomáticos por anos, apresentar apenas sintomas vagos e inespecíficos como cefaléia, ou causar distúrbios decorrentes do comprometimento de estruturas adjacentes, numa fase mais adiantada da afecção. Os tumores secretantes, geralmente são descobertos mais precocemente devido às manifestações endócrinas envolvidas.

A expansão tumoral pode ocorrer para diversas direções, resultando em manifestações neuroftálmicas de acordo com o local comprometido. O crescimento do tumor geralmente se faz em direção ascendente através do diafragma selar com comprometimento do quiasma, trato óptico e terceiro ventrículo causando perda visual progressiva. Raramente se expande pelo assoalho selar, invadindo o seio esfenoidal, de onde pode atingir a fossa nasal levando a epistaxe e obstrução nasal. A expansão para fossa craniana anterior pode acometer o lobo frontal manifestando-se com distúrbios neuropsiquiátricos como alterações de personalidade. Quando ocorre crescimento lateral do tumor, este, pode invadir a fossa craniana média ou o seio cavernoso com conseqüente comprometimento dos nervos oculomotor, troclear, abducente, trigêmio e inervação simpática ocular. O envolvimento orbitário é extremamente raro nestes tumores, com pouquíssimos casos relatados na literatura ${ }^{(5-8)}$. Karcioglu e colaboradores recentemente relataram 4 casos com invasão orbitária de adenoma hipofisário e encontraram outros 12 previamente publicados, o que evidencia a raridade desta ocorrência. Dos 16 casos previamente publicados, 8 eram prolactinomas, 5 tumores não secretantes e 1 tumor produtor de $\mathrm{TSH}^{(8)}$.

A paciente estudada representa a primeira publicada na literatura nacional e apresentava extensa lesão tumoral medindo $6,2 \mathrm{~cm} \mathrm{X} \mathrm{5,8cm} \mathrm{X} \mathrm{5,0} \mathrm{cm,} \mathrm{caracterizada,} \mathrm{portanto,} \mathrm{como}$ adenoma gigante da hipófise. Apresentava no exame de imagem massa supraselar, subtemporal e subfrontal além de componente intracavernoso e orbitário, justificando assim, os sintomas oftalmológicos por ela apresentados. Apresentava adenoma hipofisário funcionante mostrando-se com níveis alterados de GH e PRL responsáveis pelos distúrbios endócrinos como amenorréia, galactorréia e acromegalia manifesta mais tardiamente. Apesar dos distúrbios endócrinos manifestados, o diagnóstico foi feito apenas quando o tumor já se apresentava de forma bastante avançada, o que talvez fale a favor de um padrão histológico de crescimento mais agressivo e invasivo do tumor. A perda visual do OE e alteração de campo visual no OD se devem a compressão quiasmática, assim como acometimento do nervo óptico esquerdo. O quadro de oftalmoplegia completa encontrada se deve à expansão lateral do tumor com invasão do seio cavernoso, com comprometimento dos nervos cranianos que ali trafegam.

Quanto à proptose manifestada pela paciente, trata-se de uma das manifestações mais incomuns dos adenomas hipofisários. Dois são os mecanismos responsáveis por tal manifestação. O primeiro mecanismo é decorrente da oftalmoplegia, que produz um certo grau de proptose devido ao relaxamento do tônus muscular. É difícil definir com exatidão a quantidade de proptose causada por tal mecanismo. Alguns autores referem que a oftalmoplegia poderia levar a 1 a $2 \mathrm{~mm}$ de proptose $^{(9)}$, enquanto que Acers ${ }^{(10)}$ relatou um paciente que apresentava 3 $\mathrm{mm}$ de proptose decorrente de oftalmoplegia completa e que mostrou regressão completa após a recuperação do tônus motor. O segundo mecanismo, responsável pela proptose é mesmo a invasão orbitária acometendo o ápice da órbita e a fissura orbitária superior. Uma vez que o componente do tumor que invadia a órbita não era muito grande no caso em estudo, acreditamos que este não seria suficiente para explicar a magnitude da proptose $(6 \mathrm{~mm})$. O mais provável, portanto, é que tenha havido uma combinação de fatores sendo a oftalmoplegia completa responsável por parte da proptose (2 a $3 \mathrm{~mm})$ e a invasão orbitária pelo restante. Por outro lado deve ser lembrado que embora a invasão orbitária não tenha sido tão grande, esta acometia o ápice da órbita na região do canal óptico e da fissura orbitária superior, região na qual, invasões orbitárias têm, proporcionalmente, maior repercussão no deslocamento anterior do globo ocular.

$\mathrm{O}$ tratamento dos adenomas gigantes e invasivos é controverso, uma vez que a ressecção radical do tumor está associada à alta taxa de mortalidade devido a enorme extensão do tumor e com o acometimento de estruturas importantes circunvizinhas. Sendo assim, preconiza-se a ressecção parcial do tumor associada com radioterapia e tratamento clínico quando pertinente nos casos de tumores funcionantes. O prognóstico geralmente é ruim, mas existem resultados favoráveis especialmente nos tumores responsivos ao tratamento clínico, especialmente nos tumores secretantes de hormônios, como no caso em estudo.

\section{CONCLUSÃO}

Este caso se mostrou de interesse primeiramente pela raridade desta forma de expressão manifestada pelos adenomas hipofisários, uma vez que existem pouquíssimos casos relatados de acometimento orbitário por estes tumores. Nos cabe 
também salientar que existe uma variante dos adenomas hipofisários que são invasivos e que vem demonstrar que apesar do caráter histológico benigno nem sempre assumem um curso clínico favorável, fazendo-se cada vez mais importante a tentativa do diagnóstico precoce, para evitar graves seqüelas decorrentes da lesão hipofisária.

ABS TRACT

Purpose: To describe the unusual occurrence of unilateral proptosis associated with oculomotor palsy and blindness, caused by a giant pituitary adenoma. Methods: The patient was submitted to clinical and ophthalmologic examination, visual field, magnetic resonance imaging and histopathological study after surgical intervention for the removal of the tumor. Results: A 21-years-old woman had progressive bilateral visual loss, associated with proptosis and limitation of ocular motility of the left eye. Visual field examination revealed a complete temporal hemianopsia in the right eye and no light perception in the left eye. Magnetic resonance imaging showed a large tumor infiltrating the III ventricule, cavernous sinus and orbital apex. The histopathological study revealed a pituitary adenoma producing prolactin and growth hormone without signs of malignancy. Conclusions: This case is of interest not only because it documents the extremely rare occurrence of orbital involvement by a pituitary tumor but also because it emphasizes the need of an early diagnosis of invasive adenomas since such tumors not necessarily follow a benign clinical course despite their histologically benign nature.

Keywords: Exophtalmos/complications; Pituitary neoplasms/ surgery; Pituitary neoplasms/radiotherapy; Adenoma; Perimetry/methods; Blindness/etiology; Pituitary gland/pathology; Case report

REFERENCIAS

1. Anderson D, Faber P, Marcovitz S, Hardy J, Lorenzetti D. Pituitary tumors and the ophthalmologist. Ophthalmology 1983; 90:1265-70.

2. Srivastava VK, Narayanaswamy KS, Rao TV. Giant pituitary adenoma. Surg Neurol 1983;20:379-82.

3. Grote E. Characteristics of giant pituitary adenomas. Acta Neurochir 1982;60: 141-53.

4. Jefferson G. Extra sellar extension of pituitary adenomas. Proc R Soc Med 1940;33:433-58.

5. Sammartino A, Bonavolonta G, Pettinato G, Loffredo A. Exophthalmos caused by an invasive pituitary adenoma in child. Ophthalmologica 1979; 179:83-9.

6. Daita G, Yonemasu Y, Hashizume A. Unilateral exophthalmos caused by an invasive pituitary adenoma. Neurosurgery 1987;21:716-8.

7. Spiegel PH, Karcioglu ZA. Orbital invasion by pituitary adenoma. Am J Ophthalmol 1994;117:270-1.

8. Karcioglu ZA, Aden LB, Cruz AA, Zaslow L, Saloom RJ. Orbital invasion with prolactinoma: A clinical review of four patients. Ophthal Plast Reconst Surg 2002,18:64-71.

9. Migliori ME, Gladstone GJ. Determination of the normal range of exophthalmometric values for black and white adults. Am J Ophthalmol 1984; 98:438-42.

10. Acers TE. Pseudo-orbital apex syndrome. Am J Ophthalmol 1979;88:623-5.

\section{CONGRESSO INTERNACIONAL DA SOCIEDADE BRASILIENSE DE OFTALMOLOCIA} 26 e 27 de março de 2004

\section{BRASÍLIA - DF}

INFORMAÇÕES: Tel/Fax.: (61) 248-4030/8116-3304

com Dr. Hilton Medeiros ou

Tel/Fax.: (61) 248-4030/346-6971

com Ana Paula 